\title{
124 Disorders of smell
}

A Hyposmia can be caused by vasomotor rhinitis in the absence of mechanical obstruction.

B Where influenza causes a peripheral olfactory neuritis, the loss of smell is usually permanent.

C Cacosmia may be due to a foreign body in the nose.

D Temporal lobe epilepsy may cause olfactory hallucinations.

E Ammonia is used to stimulate the olfactory nerve in suspected malingerers.

\section{Cleft lip and cleft palate}

A The cleft lip results from failure of fusion of the maxillary process with the median nasal process.

B Flattening of the nostril is a feature.

C The nasal septum may be abnormally thick.

D The deformities result from teratogenic or genetic factors operating in the second month of foetal life.

E Bifid uvula is a minor form.

\section{Congenital nasal malformations}

A A midline dermoid cyst should be distinguished from a meningocoele at operation.

B A fistula is often present with dermoid cysts.

C Aplasia of the maxillary sinus is as common as aplasia of the frontal sinus.

D Exorbitism is a feature of Crouzon's syndrome.

E Atresia of the anterior nares is common in Negroes.

\section{Congenital choanal atresia}

A is most commonly a membranous closure.

$\mathrm{B}$ is most commonly bilateral.

C Occurs more often in females.

D If unilateral tends to present late with persistent watery rhinorrhea.

E Bilateral cases may be fatal.

\section{Management of maxillofacial injuries}

A The first consideration is to look for signs of shock.

$B$ The patient should be positioned supine.

C X-ray is the most useful diagnostic manoeuvre.

D Nasal obstruction indicates septal dislocation or haematoma.

E Asch's forceps are used to correct septal deformity. 